\title{
On-Line Variational Estimation of Dynamical Fluid Flows with Physics-Based Spatio-temporal Regularization
}

\author{
Paul Ruhnau, Annette Stahl, and Christoph Schnörr \\ Computer Vision, Graphics, and Pattern Recognition Group \\ Department of Mathematics and Computer Science; \\ University of Mannheim, D-68131 Mannheim, Germany \\ \{ruhnau, astahl, schnoerr\}@uni-mannheim.de \\ http: //www. cvgpr. uni-mannheim.de
}

\begin{abstract}
We present a variational approach to motion estimation of instationary fluid flows. Our approach extends prior work along two directions: (i) The full incompressible Navier-Stokes equation is employed in order to obtain a physically consistent regularization which does not suppress turbulent flow variations. (ii) Regularization along the time-axis is employed as well, but formulated in a receding horizon manner contrary to previous approaches to spatio-temporal regularization. This allows for a recursive on-line (non-batch) implementation of our estimation framework.

Ground-truth evaluations for simulated turbulent flows demonstrate that due to imposing both physical consistency and temporal coherency, the accuracy of flow estimation compares favourably even with optical flow approaches based on higher-order div-curl regularization.
\end{abstract}

\section{Introduction}

Image sequence analysis of fluid flows constitutes an active research field with a high industrial impact. Corresponding real-world measurements in concrete scenarios complement numerical results from direct simulations of the Navier-Stokes equation, particularly in the case of turbulent flows, and for the understanding of the complex spatiotemporal evolution of instationary flow phenomena. More and more advanced imaging devices (lasers, high-speed cameras, control logic, etc.) are currently developed that allow to record fully time-resolved image sequences of fluid flows at high resolutions. As a consequence, there is a need for advanced algorithms for the analysis of such data, to provide the basis for a subsequent pattern analysis, and with abundant applications across various areas.

The image measurement process proceeds as follows: First, the flow medium is seeded with small particles that are designed such that they accurately follow the fluid's motion. Next, entire velocity fields are measured by taking two or more images of the flow within short time intervals, and by estimating and interpolating the displacements of individual particles from frame to frame. This experimental method is known as Particle Image Velocimetry (PIV) [12]. Figure 1] shows a typical experimental setup in

K. Franke et al. (Eds.): DAGM 2006, LNCS 4174, pp. 444454 2006.

(C) Springer-Verlag Berlin Heidelberg 2006 
a wind tunnel. To avoid blurred images when the flow is fast, laser pulses are used. As these are only 6-10 ns long, they are capable of freezing any motion. Note that at present the described experimental setup is only capable of yielding 2D velocity fields. Therefore, we have to confine ourselves to 2D image analysis, for the time being.
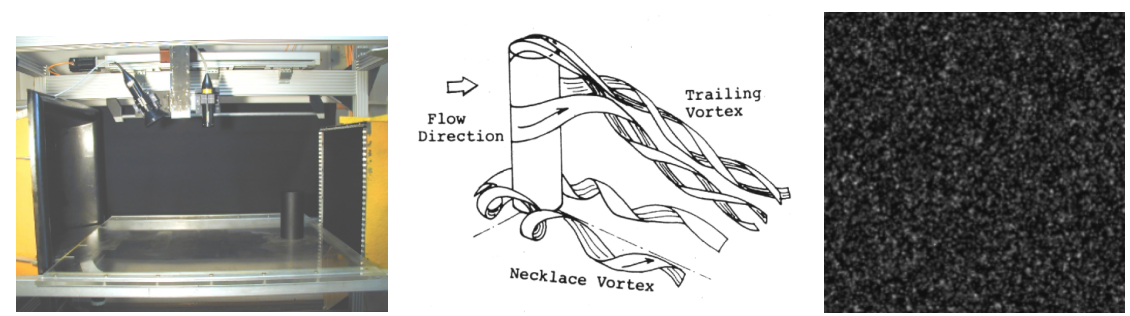

Fig. 1. Left: Experimental setting to study the flow around a cylinder. This setting results in an unsteady three-dimensional flow which can only be investigated using advanced imaging measuring techniques. Middle: Schematic illustration of typical flow phenomena [8]. Right: Typical PIV image.

A basic requirement for any motion estimation scheme is physical consistency. Otherwise, the information provided by a subsequent motion analysis is limited. Current approaches to PIV [12] do not address this issue as part of the motion estimation scheme. As a consequence, this calls for a novel combination of motion estimation and the Navier-Stokes equation which governs the real unknown flow in all applications. Our contribution in this paper is a variational approach to the estimation of motion fields constrained by the Navier-Stokes equation.

\subsection{Related Work}

Recently, variational optical flow techniques from the field of computer vision have been adopted and extended for the purpose of PIV [14|9|13/4]15]. Besides combining a carefully designed data term and coarse-to-fine estimation schemes with a standard first-order regularizer [14], a physically more plausible regularization has been suggested recently [15]. Because this approach is based on the Stokes-equation, however, it is based on related assumptions which are valid only for low Reynold numbers, i.e. nonturbulent flow. Another competitive research direction concerns the design and use of higher-order regularizers [9]4[19]. By separately penalizing the gradient of the divergence and the curl of flows, the major disadvantage of first-order regularization that penalize flow variations too much, are alleviated. Issues like well-posedness, accurated discretization and numerical stability, on the other hand, become more involved.

\subsection{Contribution}

We present a framework for fluid motion estimation that utilizes as prior knowledge that fact that flows have to satisfy the incompressible vorticity transport equation. This equation relates to the full (incompressible) Navier-Stokes equations and therefore is also valid in turbulent scenarios. Furthermore, rather than considering image pairs, our 
estimation scheme takes into account the whole image sequence. As a result, it takes into account previous estimation results so as to enforce spatio-temporal coherency and regularization, however, without penalizing flow structures that are characteristic for instationary turbulent flows. Finally, analogously to the corresponding concept from control theory, our overall algorithm works in a receding horizon manner, that is flow velocities can be computed as soon as their respective frames have been recorded. In principle, this sets the stage for the real-time extraction of instationary flow phenomena from particle image sequences.

\subsection{Organization}

We present the vorticity transport equation, which embodies our prior knowledge we use for flow estimation, in section 2.1. Section 2.2 motivates and describes our variational approach and details the resulting constrained optimization problem. Corresponding numerical issues are dealt with in section 3 Numerical experiments for evaluating the approach are presented in section 4 . We conclude in section 5.

\section{Approach}

\subsection{The Vorticity Transport Equation}

Let $u=\left(u_{1}, u_{2}\right)^{\top}, u=u(x, t), x=\left(x_{1}(t), x_{2}(t)\right)^{\top}$, denote a two-dimensional velocity field.

The incompressible vorticity transport equation is a special form of the NavierStokes equation for homogeneous flow and can be expressed as follows

$$
\frac{D \omega}{D t}=\frac{\partial}{\partial t} \omega+u \cdot \nabla \omega=\nu \Delta \omega, \quad \omega(x, 0)=\omega_{0} .
$$

It describes the evolution of the fluid's vorticity over time. Note that in the absence of external forces acting on the fluid, this equation describes the flow completely.

\subsection{Variational Model}

Let $I\left(x_{1}, x_{2}, t\right)$ denote the gray value of an image sequence recorded at location $x=$ $\left(x_{1}, x_{2}\right)^{\top}$ within some rectangular image domain $\Omega$ and time $t \in[0, T]$. We adopt the basic assumption underlying most approaches to motion estimation that $I$ is conserved. Thus, the total (material) derivative of $I$ vanishes:

$$
\frac{D I}{D t}=u \cdot \nabla I+I_{t}=0 .
$$

The spatial and temporal derivatives of $I$ of the optical flow constraint (2) are estimated locally by using FIR filters. As the focus of this paper is on physically consistent regularization and not on design of the data term, we refer the interested reader to [14] for a detailed description.

As is well known, eqn. (2) alone cannot be used to reconstruct the velocity field $u$, because any vector field with components $u \cdot \nabla I=-I_{t}$ at each location $x$ satisfies (2). 
The standard approach is to minimize the squared residual of (2) over the entire image domain $\Omega$ and to add a variational term that either enforces smoothness of the flow (first-order regularization) [17]

$$
\int_{\Omega}\left\{\left(u \cdot \nabla I+I_{t}\right)^{2}+\alpha|\nabla \cdot u|^{2}+\beta|\nabla \times u|^{2}\right\} d x,
$$

or smoothness of the divergence and vorticity (second-order regularization) [18]

$$
\int_{\Omega}\left\{\left(u \cdot \nabla I+I_{t}\right)^{2}+\alpha|\nabla(\nabla \cdot u)|^{2}+\beta|\nabla(\nabla \times u)|^{2}\right\} d x .
$$

We emphasize that both approaches (3) and (4) take only into account spatial context and determine a vector field for a fixed point in time $t \in[0, T]$.

Therefore, following the ideas of [16], our present work is an attempt to elaborate a dynamic representation of fluid flow. To this end, we solve eqn. (1) for the time interval $[0, T]$ between a subsequent pair of image frames, where $\omega_{0}$ denotes our current vorticity estimate. As a result, we obtain a transported vorticity field $\omega_{T}:=\omega(x, T)$, which can be regarded as a predicted vorticity based on the assumption that our fluid is governed by the Navier-Stokes equation. The regularization term that we employ penalizes derivations from the predicted vorticity values and forces incompressibility:

$$
\begin{gathered}
\frac{1}{2} \int_{\Omega}\left\{\left(u \cdot \nabla I+I_{t}\right)^{2}+\lambda\left(\nabla \times u-\omega_{T}\right)^{2}\right\} d x, \\
\text { s.t. } \nabla \cdot u=0 .
\end{gathered}
$$

We apply Neumann boundary conditions (i.e. $\partial u / \partial n=0$ on $\partial \Omega$ ). Note that, while the regularization term of (5) penalizes deviations between the current vorticity estimate $\omega$ and the propagated vorticity estimate of the preceding frame $\omega_{T}$, it does not enforce smoothness of the current vorticity. In practice, an implementation of (5) therefore leads to increasingly noisy vorticity estimates. Increasing the parameter $\nu$ reduces the problem only slightly: $\omega_{T}$ becomes smoother, but smoothness of $\omega$ is still not enforced directly.

To overcome this problem, we add a term that mimics the small viscous term (Laplacian) on the right-hand side of eqn. (1). Expressing the new second-order regularization term equivalently through a first-order regularizer and an additional linear constraint, we finally obtain:

$$
\begin{gathered}
E=\frac{1}{2} \int_{\Omega}\left\{\left(u \cdot \nabla I+I_{t}\right)^{2}+\lambda\left(\omega-\omega_{T}\right)^{2}+\kappa|\nabla \omega|^{2}\right\} d x \\
\text { s.t. } \nabla \cdot u=0 \\
\nabla \times u=\omega .
\end{gathered}
$$

As we usually do not have a vorticity estimate at the very first frame of an image sequence, the overall estimation process is initialized with a vorticity estimate $\omega_{0}=0$.

\footnotetext{
${ }^{1}$ It can be shown easily that the Horn\&Schunck approach [7] is just the special case of this regularization where $\alpha=\beta$.
} 
The novel vorticity transport regularizer in (6), in connection with (1), can be perceived as a special second-order div-curl regularizer: Estimated flows from a given image sequence have vanishing divergence and a curl field (vorticity) that should be smooth and as close as possible to the transported vorticity.

\section{Discretization and Optimization}

\subsection{Discretisation of the Vorticity Transport Equation}

We solve the time-dependent vorticity transport equation (1) with a second-order conservative finite difference algorithm. The method is upwind and two-dimensional in that the numerical fluxes are obtained by solving the characteristic form at cell edges (i.e. edges between adjacent pixels), and all fluxes are evaluated and differenced at the same time. The finite difference method that we employ is the Fromm-Van-Leer scheme [11].

The basic idea is to satisfy Godunov's theorem in a "natural" way. Roughly speaking, Godunov's theorem says that all methods of accuracy greater than order one will produce spurious oscillations in the vicinity of large gradients, while being secondorder accurate in regions where the solution is smooth. Accordingly, Fromm-Van-Leer's scheme detects discontinuities and adapts its behavior such that the high-order accuracy of Fromm's scheme is preserved for smooth parts of the solution, while spurious oscillations are avoided through first-order accuracy at detected discontinuities.

\subsection{Variational Approach}

For every image pair (two consecutive frames of the image sequence), we have to solve optimization problem (6) which comprises a convex functional and two linear constraint equations. We transform this constrained optimization problem into a saddle point problem. Accordingly, the unique vector field $u(x)$ minimizing (6), along with the vorticity $\omega$ and multipliers $p, q$, are determined by the variational system

$$
\begin{aligned}
a\left((u, \omega)^{\top},(\tilde{u}, \tilde{\omega})^{\top}\right)+b\left((p, q)^{\top},(\tilde{u}, \tilde{\omega})^{\top}\right) & =\left((f, g)^{\top},(\tilde{u}, \tilde{\omega})^{\top}\right), \quad \forall \tilde{u}, \tilde{\omega} \\
b\left((\tilde{p}, \tilde{q})^{\top},(u, \omega)^{\top}\right) & =0, \quad \forall \tilde{p}, \tilde{q} .
\end{aligned}
$$

The bilinear and linear forms read:

$$
\begin{aligned}
a\left((u, \omega)^{\top},(\tilde{u}, \tilde{\omega})^{\top}\right) & :=\int_{\Omega}\{u \cdot \nabla I \nabla I \cdot \tilde{u}+\lambda \omega \tilde{\omega}+\kappa \nabla \omega \cdot \nabla \tilde{\omega}\} d x, \\
b\left((p, q)^{\top},(\tilde{u}, \tilde{\omega})^{\top}\right) & :=-\int_{\Omega}\{p \nabla \cdot \tilde{u}+q(\nabla \times \tilde{u}-\tilde{\omega})\} d x .
\end{aligned}
$$

The right-hand side reads:

$$
\left((f, g)^{\top},(\tilde{u}, \tilde{\omega})^{\top}\right):=\int_{\Omega}\left\{-I_{t} \nabla I \cdot \tilde{u}+\lambda \omega_{T} \tilde{\omega}\right\} d x .
$$

We choose a regular tessellation of the image domain $\Omega$ and discretize (7) using finite elements. It is well-known from computational fluid dynamics (cf. Stokes equation) that standard first-order finite element discretizations of saddle point problems may result 

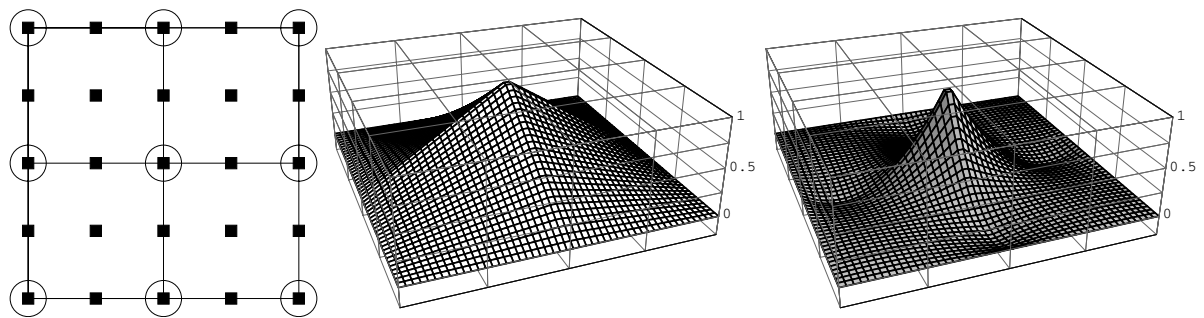

Fig. 2. Left: Sketch of 2D Taylor-Hood elements: biquadratic velocity elements (squares) and bilinear pressure elements (circles). Middle: Basis function $\phi$ of a bilinear finite element. Right: Basis function $\psi$ of a biquadratic finite element.

in instabilities or even in so-called locking effects, where the zero velocity field is the only one satisfying the incompressibility condition.

Therefore, when solving saddle point problems, mixed finite elements are traditionally used [2]. An admissible choice is the so-called Taylor-Hood element based on a square reference element with nine nodes (fig. (2). Each component of the velocity field is defined in terms of piecewise quadratic basis functions $\psi_{i}$ located at each node (the solid squares in fig. (2), whereas the Lagrange multipliers $p$ and $q$ and the vorticity $\omega$ are represented by linear basis functions $\phi_{i}$ attached to each corner node (indicated by circles in fig. (2). It can be shown that Taylor-Hood elements fulfill the so-called Babuska-Brezzi condition [2], making the discretized problem well-posed.

Indexing the velocity nodes (squares in fig. (2) by $1,2, \ldots, N$, we obtain

$$
u_{1}(x)=\sum_{i=1}^{N} u_{i} \psi_{i}(x)
$$

and similarly for $u_{2}(x)$ (where $u=\left(u_{1}, u_{2}\right)^{\top}$ ) and the components of $\tilde{u}$.

By analogy, we obtain for the $M$ Lagrange multiplier nodes (circles in fig. (2)

$$
p(x)=\sum_{i=1}^{M} p_{i} \phi_{i}(x)
$$

and similarly expressions for $q, \omega, \tilde{p}, \tilde{q}, \tilde{\omega}$. Hence, each function $u, \tilde{u}$ is represented by $2 N$ real variables, and each function $p, q, \omega, \tilde{p}, \tilde{q}, \tilde{\omega}$ is represented by $M$ real variables. For the sake of simplicity, we will use the same symbols to denote the corresponding vectors. The discretized system (7) then reads

$$
\begin{aligned}
A(u, \omega)^{\top} \cdot(\tilde{u}, \tilde{\omega})^{\top}+B^{\top}(p, q)^{\top} \cdot(\tilde{u}, \tilde{\omega})^{\top} & =(f, g)^{\top} \cdot(\tilde{u}, \tilde{\omega})^{\top}, \quad \forall \tilde{u}, \tilde{\omega} \\
B(u, \omega)^{\top} \cdot(\tilde{p}, \tilde{q})^{\top} & =0, \quad \forall \tilde{p}, \tilde{q} .
\end{aligned}
$$

These equations have to be satisfied for arbitrary $\tilde{u}, \tilde{p}, \tilde{q}, \tilde{\omega}$, thus we obtain:

$$
A\left(\begin{array}{l}
u \\
\omega
\end{array}\right)+B^{\top}\left(\begin{array}{l}
p \\
q
\end{array}\right)=\left(\begin{array}{l}
f \\
g
\end{array}\right), B\left(\begin{array}{l}
u \\
\omega
\end{array}\right)=0
$$


In order to numerically solve the saddle point problem (14), we want to employ the Uzawa algorithm (cf., e.g. [1]). However, this requires A to be positive definite which is not the case here, because the relations $u$ and $\omega$ defining $A$ in $(8)$ are mutually independent and $u$ is only involved through a degenerate quadratic form. This problem can be removed by (a) including a penalty term related to the divergence constraint into our Lagrange multiplier formulation to obtain an Augmented Lagrangian formulation [5], and by (b) splitting the vorticity matching term into two equivalent terms, one containing $\nabla \times u$ and the other one containing $\omega$. This yields the following modification of the bilinear form (8):

$$
\begin{aligned}
& a_{p}\left((u, \omega)^{\top},(\tilde{u}, \tilde{\omega})^{\top}\right):=\int_{\Omega}\left\{u \cdot \nabla I \nabla I \cdot \tilde{u}+\frac{\lambda}{2}(\omega \tilde{\omega}+(\nabla \times u)(\nabla \times \tilde{u}))\right. \\
& +\mu(\nabla \cdot u)(\nabla \cdot \tilde{u})+\kappa \nabla \omega \cdot \nabla \tilde{\omega}\} d x .
\end{aligned}
$$

We point out that this modification is done for numerical reasons only. It does not change the optimization problem (6). Matrix $A_{p}$ resulting from the discretization of (15) is positive definite and, because $u$ and $\omega$ do not explicitly depend on each other, can be split into two systems:

- The system containing $u$ is the linear system with a simple first-order div-curl regularization (cf., e.g. [17], and (3)).

- The system containing $\omega$ corresponds to a simple first-order quadratic functional.

Because $A_{p}$ is invertible and well-conditioned, we solve the first equation of the system (14), with A replaced by $A_{p}$, for the unknown $u$

$$
\left(\begin{array}{l}
u \\
\omega
\end{array}\right)=A_{p}^{-1}\left[\left(\begin{array}{l}
f \\
g
\end{array}\right)-B^{\top}\left(\begin{array}{l}
p \\
q
\end{array}\right)\right],
$$

and insert the result into the second equation:

$$
B A_{p}^{-1}\left[\left(\begin{array}{l}
f \\
g
\end{array}\right)-B^{\top}\left(\begin{array}{l}
p \\
q
\end{array}\right)\right]=0 .
$$

This problem only involves the adjoint variables $p, q$ :

$$
\left(B A_{p}^{-1} B^{\top}\right)\left(\begin{array}{l}
p \\
q
\end{array}\right)=B A_{p}^{-1}\left(\begin{array}{l}
f \\
g
\end{array}\right) .
$$

The matrix $\left(B A_{p}^{-1} B^{\top}\right)$ is symmetric and positive definite. Therefore, we apply the conjugate gradient iteration to (18). This requires a single matrix inversion in every iteration step. This is efficiently accomplished using multi grid iteration (cf. [6]).

\section{Experimental Evaluation}

This section shows numerical results on ground truth fluid image sequences obtained with our approach in comparison with first-order regularization and with second-order div-curl regularization. 

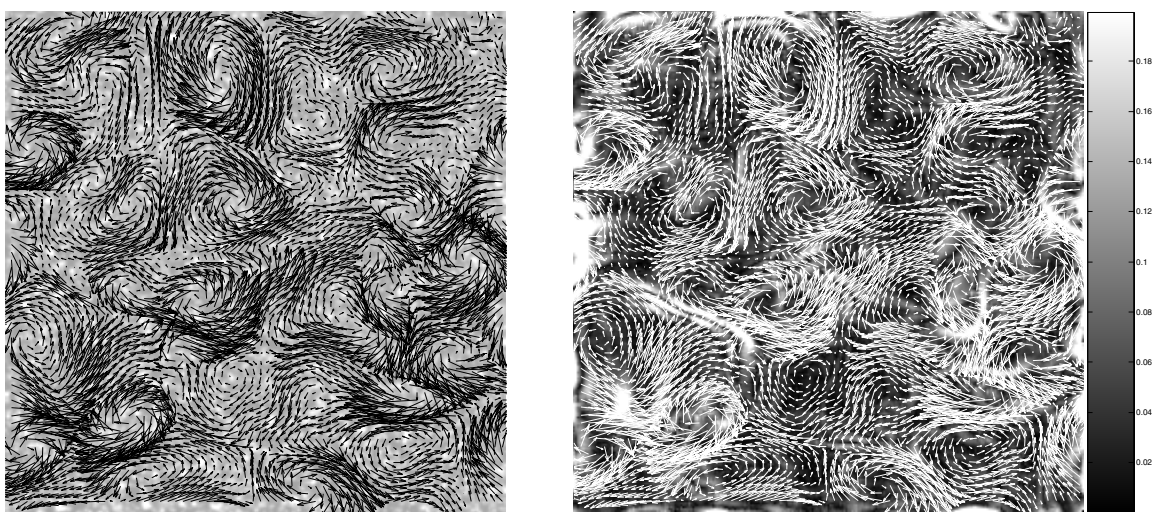

Fig. 3. Left: 100th frame of the synthetic image sequence with ground truth velocity field. Right: Estimated velocity field for the 100th frame. The background intensity shows the absolute RMS error (brighter $=$ larger error), which is about $0.055 \mathrm{px}$. on average (cf. fig. 4).

The evaluation of our approach from the viewpoint of fluid mechanics (real data, without ground-truth) is beyond the scope of this paper.

The synthetic PIV image sequence that we used for testing was provided by [3]. The underlying velocity field was computed by a so-called pseudo-spectral code that solves the vorticity transport equation in Fourier space and evaluates a subgrid model for simulating small-scale turbulent effects on the larger scales of the flow. These latter effects, of course, are not known in practice, nor was anything related to that used while evaluating our approach.

In order to simulate the intensity function of real PIV images, the computed velocity fields are used to transport collections of (images of) particles that are typically used for the seeding of flows so as to make them visible (cf. section 1). The scheme resembles the one described in [10]. We used the first 100 frames of the synthesized PIV image sequence and compared the following three approaches:

- Horn\&Schunck [7]: First-order regularization, temporal coherency is not exploited, no incompressibility constraint is imposed. The smoothness parameter $\lambda=0.005$ was manually selected for best performance.

- 2nd Order Regularization [19]: These authors used higher-order regularization with an additional incompressibility constraint. Instead of mixed finite elements (as we do), the authors used the so-called mimetic finite differencing scheme. Temporal coherency is not exploited. Parameters: $\lambda_{1}=0.5, \lambda_{2}=0.05$, manually selected for best performance.

- Vorticity Transport Approach (this paper): As described above, higher-order regularization is used, the incompressibility constraint is imposed, and temporal coherency is exploited in an on-line manner. Parameters $\lambda=0.005, \mu=0.005$, $\nu=0.1, \kappa=0.0005$. As for the other approaches, we selected the regularization parameters $\lambda, \mu, \kappa$ by hand. Note that the viscosity coefficient $\nu$ is not a free user parameter but characterizes the physical nature of the fluid flow. 


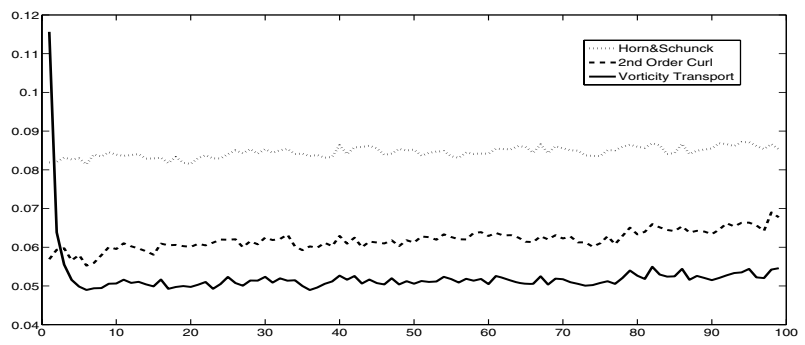

Fig. 4. Average absolute RMS error (in pixels) for frames 1-100, using three different methods. First-order regularization performs worse than second-order regularization. Both error curves are constant because temporal coherency is not exploited. The approach based on vorticity transport starts with a rather low accuracy (assumption of $\omega=0$, which is not valid) but then becomes significantly more accurate than the two other techniques due to the physically consistent regularization over time. This novel spatio-temporal regularization is achieved with an on-line computational scheme and fixed storage requirements, irrespective of the length of the image sequence. The decay of the error curve within the first 10 frames clearly displays the usage of this implicitly encoded "memory".
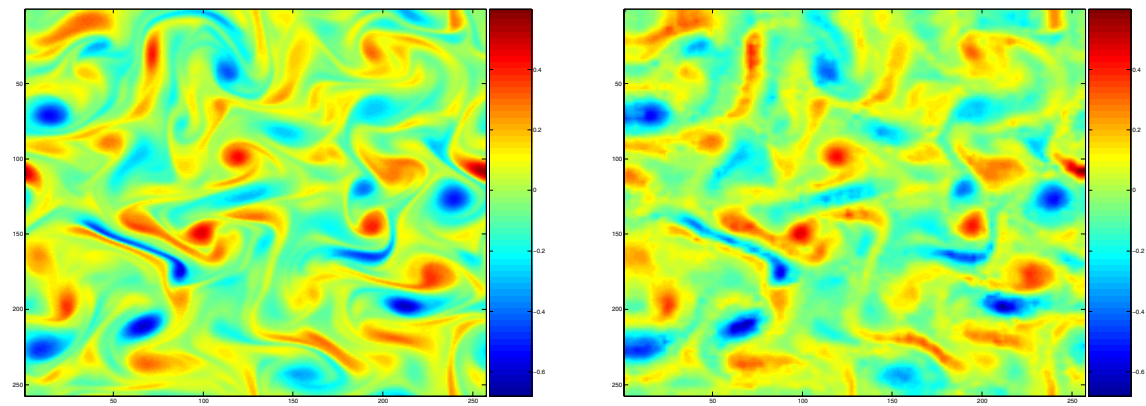

Fig. 5. Left: True vorticity of frame 100. Lower Right: Estimated vorticity $\omega$ for frame 100. For the first frame, the estimation process was initialized with $\omega=0$, corresponding to "nothing is known in advance". The result on the right shows that not only the vorticity transport equation has been successfully adapted to the observed image sequence, but that it improves the accuracy of flow estimation in terms of $u$, too (cf. fig. (4). As a consequence, flow derivatives can be estimated fairly accurate, as shown in the right panel. Such quantitative information is very important in connection with imaging-based experimental fluid mechanics.

Figure 4 compares the errors of all three approaches over time. The first-order regularization approach yields the highest errors, while the second-order approach is much more accurate. The errors of both approaches stay constant over time because each subsequent image pair is independently evaluated and temporal coherency is ignored.

For the first frame, the approach presented in this paper, utilizing the vorticity transport equation, shows worse performance than the other two algorithms. During the subsequent period of time, however, the error of the vorticity transport approach de- 
creases considerably, because not only higher-order regularization is used but temporal coherency is successfully exploited as well.

We emphasize that temporal coherency does not mean smoothness. Rather, the flow exhibits high spatio-temporal gradients as turbulent fluids do. Temporal coherency relates to a physically consistent transport mechanism interacting with flow estimation from an image sequence. Due to the on-line computational scheme, fixed computational resources are needed no matter how long the image sequence is. The decay of the error curve over several frames in figure 4 shows, however, that the approach is able to memorize the history longer than just the previous frame.

Figure 3 displays the estimated velocity for the for the 100th frame, along with the respective RMS errors. The reconstructed velocity field is surprisingly exact, in view of the highly non-rigid motion we are dealing with. Figure 5 shows that even the vorticity related to flow derivatives is reconstructed quite well under these difficult conditions. We expect such quantitative data to be valuable information in connection with imaging-based fluid mechanics.

\section{Conclusion}

We presented an approach to fluid motion estimation that uses the vorticity transport equation for physically consistent spatio-temporal regularization. The approach combines variational motion estimation with higher-order regularization and motion prediction through a transport process. For motions that conform to our assumption (i.e. fluids that are governed by the incompressible 2D Navier-Stokes equation), a temporal regularization effect, computed in a recursive manner, was demonstrated. In these scenarios, our approach outperforms advanced variational approaches for optical flow estimation.

\section{Acknowledgment}

Support by the Deutsche Forschungsgemeinschaft (DFG, SCHN 457/6) within the priority programme "Bildgebende Messverfahren in der Strömungsmechanik" (www . spp1147.tu-berlin.de) and by the EU-project "Fluid Image Analysis and Description" (http: / fluid.irisa.fr/) is gratefully acknowledged.

\section{References}

1. D. Braess. Finite elements. Theory, fast solver \& appl. in solid mechanics. Springer, 1997.

2. F. Brezzi and M. Fortin. Mixed and hybrid finite element methods. Springer-Verlag New York, Inc., New York, NY, USA, 1991.

3. J. Carlier and D. Heitz. 2D turbulence sequence provided by Cemagref within the European Project 'Fluid Image Analysis and Description'.

4. Th. Corpetti, D. Heitz, G. Arroyo, E. Mémin, and A. Santa-Cruz. Fluid experimental flow estimation based on an optical-flow scheme. Exp. Fluids, 40(1):80-97, 2005.

5. M. Fortin and R. Glowinski. Augmented Lagrangian Methods: Applications to the Numerical Solution of Boundary-value Problems. North-Holland, Amsterdam, 1983. 
6. W. Hackbusch. Iterative Solution of Large Sparse Systems of Equations, volume 95 of AMS. Springer, 1993.

7. B. Horn and B. Schunck. Determining optical flow. Art. Intelligence, 17:185-203, 1981.

8. T. Kawamura, M. Hiwada, T. Hibino, I. Mabuchi, and M. Kumada. Flow around a finite circular cylinder on a flat plate. Bulletin of the JSME, 27(232):2142-2151, 1984.

9. T. Kohlberger, E. Mémin, and C. Schnörr. Variational dense motion estimation using the helmholtz decomposition. In L.D. Griffin and M. Lillholm, editors, Scale Space Methods in Computer Vision, volume 2695 of LNCS, pages 432-448. Springer, 2003.

10. K. Okamoto, S. Nishio, and T. Kobayashi. Standard images for particle-image velocimetry. Meas. Sci. Technol., 11:685-691, 2000.

11. E. G. Puckett and P. Colella. Finite Difference Methods for Computational Fluid Dynamics (Cambridge Texts in Applied Mathematics). Cambridge University Press, 2005.

12. M. Raffel, C. Willert, and J. Kompenhans. Particle Image Velocimetry. Springer, 2001.

13. P. Ruhnau, C. Gütter, and C. Schnörr. A variational approach for particle tracking velocimetry. Meas. Sci. Technol., 16(7):1449-1458, 2005.

14. P. Ruhnau, T. Kohlberger, H. Nobach, and C. Schnörr. Variational optical flow estimation for particle image velocimetry. Exp. Fluids, 38:21-32, 2005.

15. P. Ruhnau and C. Schnörr. Optical stokes flow: An imaging based control approach. Exp. Fluids, 2006. submitted.

16. A. Stahl, P. Ruhnau, and C. Schnörr. A distributed-parameter approach to dynamic image motion. In Int. Workshop on The Repres. and Use of Prior Knowl. in Vision, LNCS, Springer, ECCV 2006. in press.

17. D. Suter. Mixed finite elements and whitney forms in visual reconstruction. In Geometric Methods in Computer Vision II, pages 51-62, 1993.

18. D. Suter. Mixed-finite element based motion est. Innov. Tech. Biol. Med., 15(3), 1994.

19. J. Yuan, P. Ruhnau, E. Mémin, and C. Schnörr. Discrete orthogonal decomposition and variational fluid flow estimation. In Scale-Space 2005, volume 3459 of Lect. Not. Comp. Sci., pages 267-278. Springer, 2005. 